\title{
A comparison of the randomization test with the $F$ test when error is skewed
}

\author{
D. J. K. MEWHORT \\ Queen's University, Kingston, Ontario, Canada
}

\begin{abstract}
I compared the randomization/permutation test and the $F$ test for a two-cell comparative experiment. I varied (1) the number of observations per cell, (2) the size of the treatment effect, (3) the shape of the underlying distribution of error and, (4) for cases with skewed error, whether or not the skew was correlated with the treatment. With normal error, there was little difference between the tests. When error was skewed, by contrast, the randomization test was more sensitive than the $F$ test, and if the amount of skew was correlated with the treatment, the advantage for the randomization test was both large and positively correlated with the treatment. I conclude that, because the randomization test was never less powerful than the $F$ test, it should replace the $F$ test in routine work.
\end{abstract}

Because computation is becoming less expensive, statistical techniques using a desktop computer are now possible that would have required a supercomputer a decade ago. An example is the randomization test, a computationally intensive alternative to the $F$ or $t$ test. One advantage of the randomization test is that it does not depend on a particular distribution for error, and unlike nonparametric tests based on a transformation to ranks, it uses all the information in the data (see Bradley, 1968).

The present article compares the power of the randomization test with that of the $F$ test with both normal and skewed error. Skewed error distributions are common in experiments that use dependent measures based on time or correlates of time such as distance traveled in a water maze. The randomization test is described by Edgington (1995) and by Manly (1997); applications to standard experiments (in animal behavior) and specialized data analysis (in brain imaging) are described by Mundry (1999) and by Nichols and Holmes (2001), respectively. Because the randomization test may be unfamiliar, however, the first section of this article outlines the logic of the randomization test.

The research was supported by grants from the Natural Sciences and Engineering Research Council of Canada and by an Academic Equipment Grant from Sun Microsystems of Canada. I also acknowledge assistance of the High Performance Computing Virtual Laboratory for parallel computing infrastructure and support. This article is based on a report read at the annual meeting of the Canadian Society for Brain, Behaviour, and Cognitive Science, St. John's, Newfoundland, June 2004. I am indebted to Andrew Heathcote, Scott Brown, Randy Jamieson, Bill Hockley, Ben Murdock, and Beth Johns for comments on an earlier draft and to Darren Popham for assistance in writing the code. Correspondence should be addressed to D. J. K. Mewhort, Department of Psychology, Queen's University, Kingston, ON, K7L 3N6 Canada (e-mail: mewhortd@post.queensu.ca).

Note-This article was accepted by the previous editor, Jonathan Vaughan.

\section{THE RANDOMIZATION TEST}

Consider a simple comparative experiment: Depending on the topic, the units of analysis - the subjects may be plots of land in the study of a fertilizer's effect on the growth of grain, pigs in a study of a drug's action on blood pressure, or undergraduates in a study of retrieval from memory. Although the subjects are selected to be as uniform and as representative as possible — animal subjects are often obtained from a commercial breeding facility to limit genetic variability - they are inherently variable; that is, some yield higher scores than others for reasons unrelated to the topic of investigation. Furthermore, the differences among the subjects (noise) are sufficiently large that they may mask the effect that the experimenter wishes to study.

Given that subject-to-subject variability may mask the effects of interest, experimenters study groups of subjects instead of basing inferences on data from individual subjects. Specifically, the experimenter selects a suitable set of subjects and divides them at random into two groups. One group receives one treatment, and the other receives a placebo; but, of course, the comparison may involve any two treatments. It is common practice is to label groups the "experimental" and "control," respectively. A measurement is taken from each subject, and the difference in the means for the two groups is taken as a measure of the treatment's effect.

If the treatment has no effect, the group means reflect the (random) distribution of scores to the cells. The experimenter's problem is to decide whether the difference between the means of the two groups reflects the treatment or just noise. Statistical analysis enlists probability theory to deal with the problem.

By assigning the subjects to the cells at random, the experimenter has defined a chance model for the exper- 
iment. If the treatment has no effect (a proposition known as the null hypothesis), the difference between the two averages must reflect subject-to-subject variability. Before the groups were formed, however, each subject had an equal chance of being assigned to the experimental group. That is, when the subjects were assigned to the cells, the experimenter picked one assignment at random from the set of all possible assignments.

To apply the chance model, the experimenter counts the number of outcomes that would have yielded a difference in the means as extreme as the outcome that was actually obtained. To do so, the experimenter must first choose an appropriate comparison measure for the two cells, such as the difference in means, and then calculate what the difference in the comparison measure would have been under each of the possible ways that the subjects could have been assigned to the cells. If the number is smaller than the number of possible outcomes, the experimenter either has to accept that (1) the difference reflects noise and that chance has played an unlikely trick or (2) the effect of the treatment is real. It is conventional to reject the first option in favor of the second if the number represents less than $5 \%$ of the possible outcomes. The test leading to the choice is called a test for significance, and that conducted by considering all of the possible outcomes is called a randomization test (Box \& Andersen, 1955; Pitman, 1937).

A randomization test is tedious to compute by hand. If the two cells each contain 10 subjects, for example, there are $C(20,10)=20 ! /(10 ! 10 !)=184,756$ combinations to consider. ${ }^{1}$ Hence, even though the chance model maps directly onto the procedure used to conduct the experiment - a procedure common in agricultural, biomedical, industrial, and psychological experimentsthe randomization test is not popular in routine work. Instead, researchers compute a test based on a chance model first proposed by C. F. Gauss (1777-1855) as part of an analysis of measurement error (see Stigler, 1986).

When one uses Gauss's theory for measurement error, each score in the experiment can be treated as a sum of two parts, a true value that reflects the conditions common to the subjects within a cell, plus an error value that reflects the individual subject at the time the measurement was taken. Because the subjects were randomly assigned to the cells, the two sets of scores can be treated as random samples from two distributions, each defined by the cell's condition, combined with measurement error. That is, the population refers to the population of measurements defined by the apparatus, subjects, and measurement procedures used in the experiment. Under the null hypothesis, the cells are not different; hence, the two sets of scores can be treated as samples from a single distribution. A test for significance can be computed using statistical sampling theory to estimate the probability that the two are samples from the same distribution of error.

A test for significance based on sampling theory and the Gauss model for error usually requires the calcula- tion of only the mean and variance for the two cells, and, for that reason, the arithmetic is easier to compute than that for a randomization test. The fly in the ointment is that the test's derivation requires the experimenter to define the distribution of measurement error. Gauss used the normal distribution to describe measurement error, and experimenters routinely rely on Gauss's invention in tests for significance. Whether measurement error is well described by the normal distribution remains an open question. According to Gabriel Lippmann (1908 Nobel laureate in physics), empiricists accept it because they think it is a mathematical theorem, and mathematicians accept it because they think it is an experimental fact (cited by Freedman, Pisani, Purves, \& Adhikari, 1991, p. 282).

In practice, experimenters usually accept the normal distribution as a description of error without subjecting their assumption to close scrutiny. Some procedures can detect violations of the normal form, but they are usually ineffective unless the number of observations is large. Moreover, a good deal is known about the performance of tests based on the normal theory of error when the data are clearly inconsistent with that theory. As a general rule, modest violations of the assumption are not too serious. To assess the effect of violating an assumption, it is customary to conduct a Monte Carlo study, in which the test is computed using artificial data that exhibit the particular violation of normal theory, and the test's performance under the violation is compared with the case using data that are consistent with normal theory.

Because the chance model used in a randomization test is based on the distribution of experimental units to conditions, the logic of the randomization test does not assume a particular distribution of error. If the experiment has been conducted properly - that is, if the subjects have indeed been randomly assigned to the conditionsa randomization test is valid, regardless of the underlying form of error. Nevertheless, it likely works best when error has the properties that Gauss built into his account of measurement error: Small errors are more frequent than large ones, and the distribution of errors is symmetrical, so that the mean of the measurements is not biased systematically (Hoeffding, 1952).

The chance model used in a randomization test is based on an action carried out in a particular experimentnamely, the experimenter's assignment of units to conditions. Because each test is local to a particular experiment, it does not lend itself to analytic mathematical analysis, and, perhaps for that reason, little is known about how the underlying distribution of error affects performance of the randomization test as a general procedure (but see Baker \& Collier, 1966; Keller-McNulty \& Higgins, 1987; Kempthorne \& Doerfler, 1969; Romano, 1990). The next sections compare the randomization and $F$ tests when error is normal and when it is skewed, first when skew is not correlated with the treatment and, then, when it is correlated with the treatment. 


\section{Normal Error}

The case with normal error is of interest for historical reasons. In his classic text, Design of Experiments, Fisher (1935/1966) computed a randomization test on data from Charles Darwin and compared the results with those computed from the same data using a normal-error test. He found substantially the same results and used the example to argue for the validity of tests based on normal error. In his words, because randomization tests "assume less knowledge, or more ignorance, of the experimental material than do the standard tests" (p. 47), they provide the means with which to assess an experiment when there is reason to believe that "simpler tests may have been injured by departures from normality" (p. 48).

In the first Monte Carlo study, I generated data for two cells using a random number generator adapted from software by Press, Teukolsky, Vetterling, and Flannery (1992, ran3, pp. 267-274), combined with a normaldeviate filter (gasdev, pp. 279-280). I selected parameters for the data so that the sensitivity of the tests would cover a full range - that is, so that the probability of rejecting the null hypothesis at the $5 \%$ level of confidence would range from .05 with a null treatment to 1 with a large separation between the means. Specifically, I set the mean $(m u)$ of the normal generator at 400 and the standard deviation (sigma) at 60.

For each set of artificial data, I computed both a randomization test and an $F$ test and recorded the resulting probability values. To compute the randomization test, I calculated the absolute value of the difference between the means for each possible assignment of subjects to conditions and tallied the number of outcomes as extreme or more so than the obtained (initial) outcome. The resulting probability was defined by the ratio of the number of extreme cases to the number of possible assignments. To compute the $F$ test, I calculated the $F$ ratio for obtained outcome and calculated the associated probability using routines recommended by Press et al. (1992, pp. 219-223).

I varied the number of subjects per cell $(n)$ from 4 to 10 , and the experimental and control cells were equal in number - that is, $n_{1}=n_{2}$. I manipulated the size of the treatment effect by adding an appropriate constant to the experimental cell, but not to the control cell. I varied separation between the means from 0 to 150 in 11 steps (i.e., from 0 to $2.5 \times$ the standard deviation of the population defined by the random-number generator). Finally, I repeated the exercise 10,000 times for all combinations of $n$ and of the separation between the means.

Figure 1 summarizes the results. The top panel shows a family of sensitivity curves obtained by calculating the proportion of times that the randomization test rejected the null hypothesis at the 5\% level of significance as a function of the number of observations per cell and the separation between the means. The middle panel shows the corresponding data using the $F$ test instead of the randomization test. The bottom panel plots the difference between the two tests. A positive difference indicates an

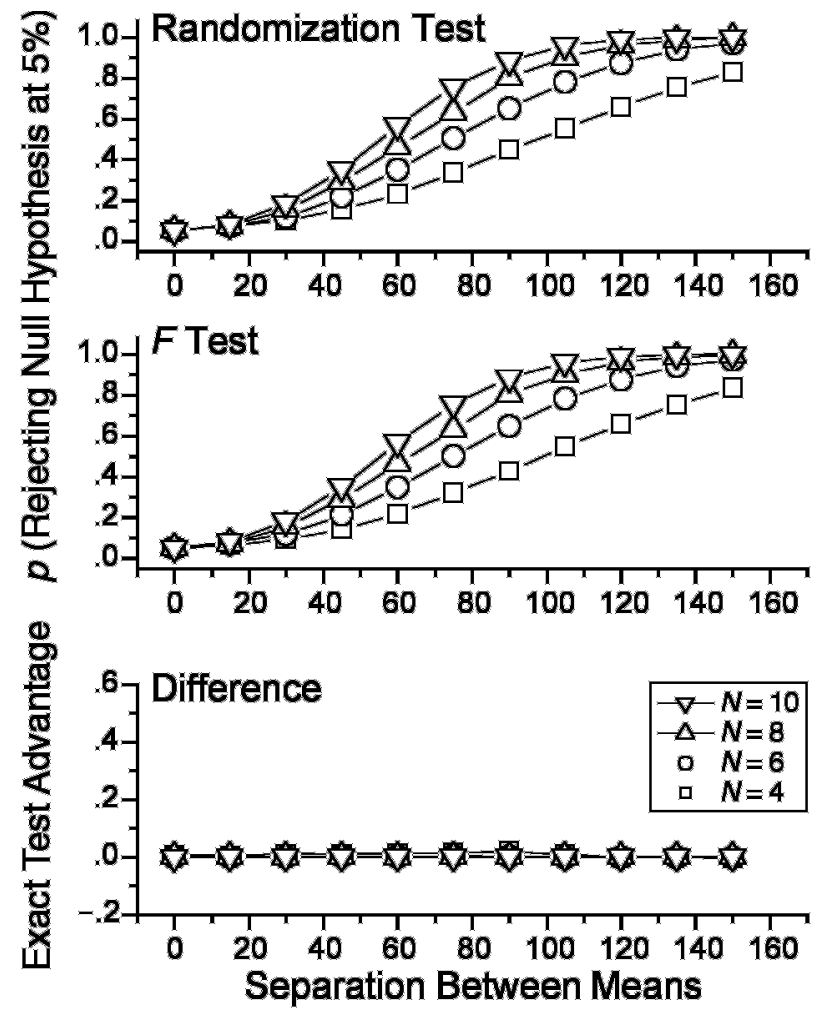

Figure 1. Monte Carlo comparison of the between-subjects randomization test with the $F$ test, using normal error. The top and middle panels show the probability of rejecting the null hypothesis at the $5 \%$ level of confidence as a function of the number of observations per cell and the effect size for the randomization and $F$ tests, respectively. The bottom panel shows the difference between the two tests.

advantage for the randomization test, whereas a negative difference indicates an advantage for the $F$ test.

As shown in Figure 1, when the null hypothesis was true, both the randomization test and the $F$ test rejected the null hypothesis about $5 \%$ of the time. The probability of detecting the effect increased systematically both as the separation between the means was increased and as the number of observations per cell was increased. With $n=8$ or more, both tests detected an effect of about twice sigma almost all the time. There was no systematic advantage for one test over the other.

As Fisher (1935/1966) anticipated, when the underlying error was normal, the $F$ and randomization tests provided essentially the same results. The conclusion must be qualified, however. With $n=2$, there are only $\mathrm{C}(4,2)=$ 6 possible outcomes. Hence, for a randomization test with $n=2$, not even the most extreme outcome can be rare enough to reject the null hypothesis at the $5 \%$ level of confidence. It is possible, however, to reject the null hypothesis using the $F$ test, because the assumption that error has a normal distribution provides additional information. The extra information, in turn, underwrites the extra sensitivity that allows the null hypothesis to be rejected. With 
$n=5$, there are 252 ways in which to assign subjects to the cells, and that number is large enough to give the randomization test sensitivity that is close to the $F$ test.

\section{Skewed Error}

In some standard experiments, error can be expected to deviate from normal. In studies of human choice, perception, and recognition memory, for example, decision latency (in milliseconds) is often taken to be a measure of the difficulty of the task. In animal studies, response time (RT) or a measure correlated with time, such as swimming distance in a water maze, plays an equivalent role. In both cases, the minimum value of the measure is bounded by factors unrelated to the task's difficulty (e.g., a minimum time or minimum distance to respond), but the maximum is open ended. Because one end of the scale has a bound but the other end is unbounded, the empirical distributions of scores are often skewed.

However, experimental evidence suggests that another factor contributes to the skew, because the amount of skew is sometimes connected to the difficulty of the decision itself and sometimes not. A good example is provided by Hockley's (1984) detailed analysis of Sternberg's (1969) classic study of recognition memory. Hockley had subjects study a series of 3-6 items, and they were then asked to indicate whether or not a probe item was a member of the study set. Mean decision time increased as the number of items in the study set was increased. Importantly, the increase in mean decision time reflected a corresponding increase in the skew of the distribution of RTs (Hockley's findings have been confirmed independently by Ashby, Tein, \& Balakrishnan, 1993). A similar pattern occurs for retrieval of information from memory in semantic-comparison tasks. For example, the time to verify a digit's value, relative to a standard, increases with its distance from the standard (e.g., Link, 1990; Moyer \& Landauer, 1967). Smith and Mewhort (1998) have shown that the increase in mean verification time reflects a corresponding increase in the skew of the underlying distributions of decision times; that is, the skew is correlated with the decision's difficulty.

Although the amount of skew is sometimes associated with the difficulty of the decision, that pattern is not universal: Hockley (1984) did not find an increase in skew when subjects were asked to search visual displays that contained an increasing number of foils. Instead, increasing the number of foils in the display increased mean decision time by translating the whole distribution to the right. Translating the distribution is the pattern explored in the normal-error data in Figure 1, and it is the pattern anticipated by standard statistical models, such as the analysis of variance.

On Hockley's (1984) evidence, then, changes in skew may occur with some manipulations, but not with others, even though the two cases share the same response measure (decision latency) and a number of other characteristics. Using decision time as a measure of difficulty in a choice experiment, one can anticipate that the distribution of error is skewed to the right, but it is difficult to anticipate whether a particular manipulation will increase skew (as in Hockley's retrieval-from-memory experiment) or translate the cell's distribution to the right (as in Hockley's visual-search experiment). From an experimenter's perspective, it is difficult to know into which class a particular manipulation will fall until after the fact. For that reason, it is important to understand the operating characteristics of the statistical tools for both situations, and, in particular, the difference between tests that use a chance model based directly on random assignment versus those that rely on the Gaussian analysis of error.

Skew uncorrelated with the treatment effect. The general procedure was the same as before. To generate skewed error instead of normal error, however, I used the ex-Gaussian distribution instead of the normal distribution.

The ex-Gaussian distribution is defined by the convolution of a normal distribution with an exponential distribution. It has three parameters, $m u$, sigma, and tau; $m u$ and sigma are the mean and standard deviation of the parent normal distribution, whereas tau is the mean of the parent exponential distribution. When $\operatorname{tau}=0$, the ex-Gaussian distribution collapses to the normal; hence, tau is a skew parameter.

I used the ex-Gaussian distribution for two main reasons. First, it is widely used to describe the distribution of latency scores in the analysis of decision and choice (e.g., Heathcote, Brown, \& Mewhort, 2002; Hockley, 1984; Ratcliff \& Murdock, 1976) and therefore, provides a good facsimile of real data. Second, the ex-Gaussian has convenient properties: The mean of an ex-Gaussian distribution equals $(m u+t a u)$, and its variance equals $\left(s i g m a^{2}+t a u^{2}\right)$. For the Monte Carlo comparison, I set $m u$ to 400 , sigma to 60 , and, in separate analyses, tau to either 100 or 200 . The parameters are typical of experimental data from human judgment tasks (e.g., Heathcote, Popiel, \& Mewhort, 1991; Smith \& Mewhort, 1998).

I manipulated the size of effect in 11 steps from 0 to 400 by adding the treatment effect to the $m u$ parameter. By doing so, I implemented a translation effect of the sort that Hockley (1984) found in visual search. I used a larger range for the treatment than in the normal-error condition so that the sensitivity of the tests would cover the full range.

The results are summarized in Figure 2. The top panels show a family of sensitivity curves obtained by calculating the proportion of times that the randomization test rejected the null hypothesis at the $5 \%$ level of confidence as a function of $n$ and of the separation between the means. The middle panels show the corresponding data using the $F$ test. Finally, the bottom panels show the difference between the two tests when applied to the same data. The panels in the left column reports the data when tau was set to 100 , and these in the right column report the data when tau was set to 200 .

As is shown in Figure 2, the general pattern of results is similar to that in the normal-error case. When the null hypothesis was true, both the randomization and $F$ tests 

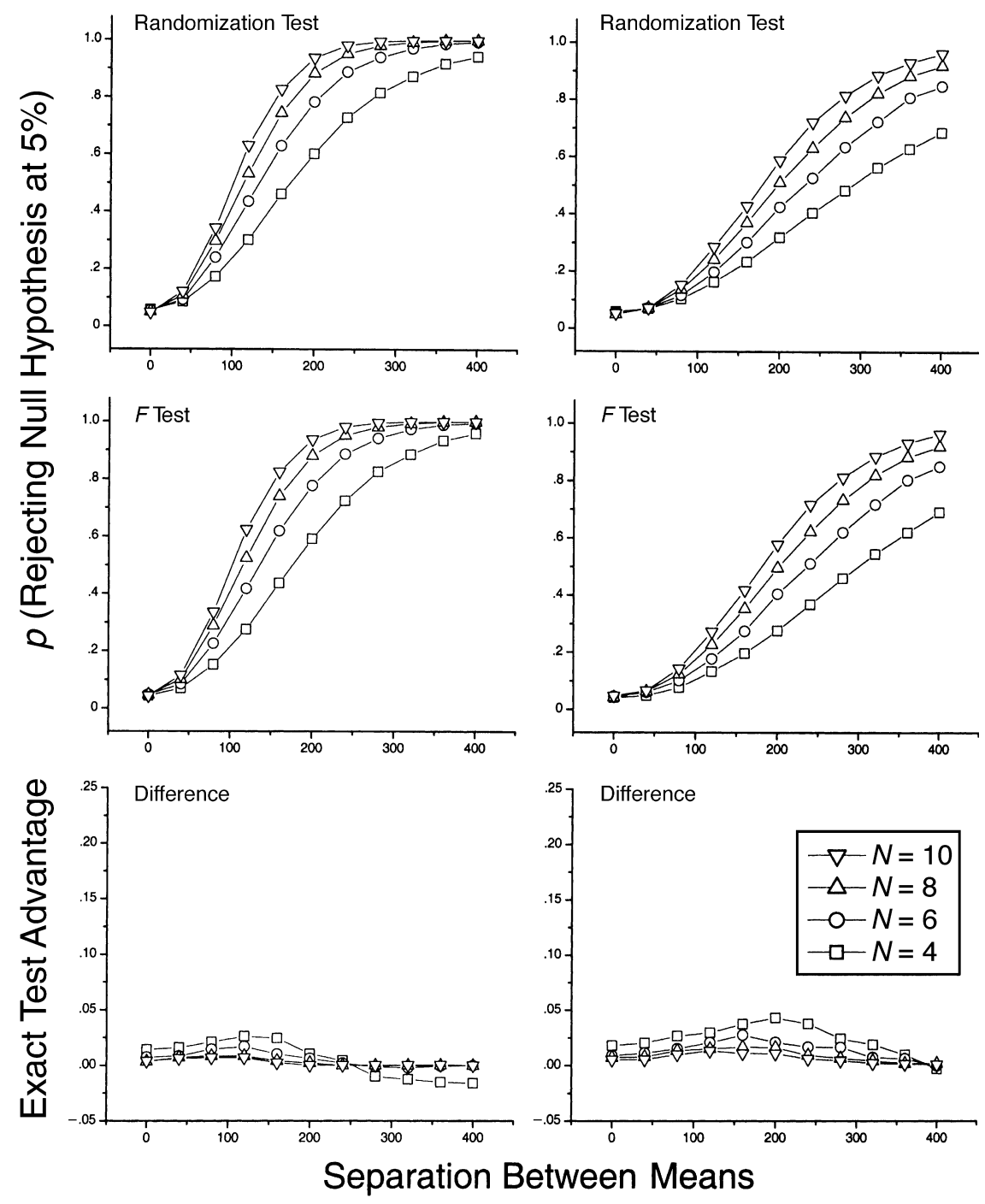

Figure 2. Monte Carlo comparison of the between-subjects randomization test and the $F$ test, using skewed error; the skew is uncorrelated with the treatment. The top and middle panels show the probability of rejecting the null hypothesis at the $5 \%$ level of confidence as a function of the number of observations per cell and effect size for the randomization and $F$ tests, respectively. The bottom panels show the difference between the two tests. The panels in the left column show the data with a modest skew, whereas those in the right column show the data with a large skew.

rejected the null hypothesis about $5 \%$ of the time. The probability of detecting the treatment's effect increased systematically as both its size and $n$ increased. Unlike the normal-error condition shown in Figure 1, however, the randomization test was slightly more likely to reject a false null hypothesis than was the corresponding $F$ test. The advantage in sensitivity was greater when the skew was large and when the $n$ was small.

Finally, comparison of the normal-error case shown in Figure 1 showed the overall sensitivity of both tests to be dramatically worse. Skew in the error distribution reduced the probability of detecting a true treatment effect. For example, with a separation of the means at 150 with normal error (Figure 1), both tests detected the differ- ence about $80 \%$ of the time with only four observations per cell. With skewed error (Figure 2), both tests detected the same separation between the means about $60 \%$ of the time with 10 observations per cell.

The conclusion, so far, is that skewed error reduces the sensitivity of both tests more than in the normal-error case. A slight sensitivity advantage favored the randomization test when error was skewed, but the advantage was small, perhaps too small to prompt a researcher to abandon the familiar $F$ test in favor of the less familiar randomization test.

Skew (and variance) correlated with the treatment. Next, I considered the case in which the treatment is correlated with skew in the error distribution, the pattern 
that Hockley (1984) found for short-term recognitionmemory experiments. As before, I used an ex-Gaussian distribution with $m u=400$, sigma $=60$, and tau $=100$ to generate skewed error distribution, and to allow the sensitivity to cover the full range, I manipulated the treatment effect (i.e., the separation between the means) over the 0-600 range, a larger range than before. In contrast to the earlier examples, however, the treatment was manipulated by increasing the skew of the experimental cell rather than by translating the experimental distribution. Increasing the skew also increased the variance of the experimental cell. In terms of the ex-Gaussian distri- bution, the treatment was implemented by increasing tau instead of $m u$.

The left panels of Figure 3 summarize the results. As shown in the top two panels, both tests rejected a true null hypothesis about $5 \%$ of the time, and increasing both the separation between the means and the number of observations per cell increased the probability of detecting the difference. In contrast with the case with skew uncorrelated with the treatment, however, a large and systematic advantage favored the randomization test over the $F$ test (shown in the bottom panels). For the cases considered here-which are typical of those found
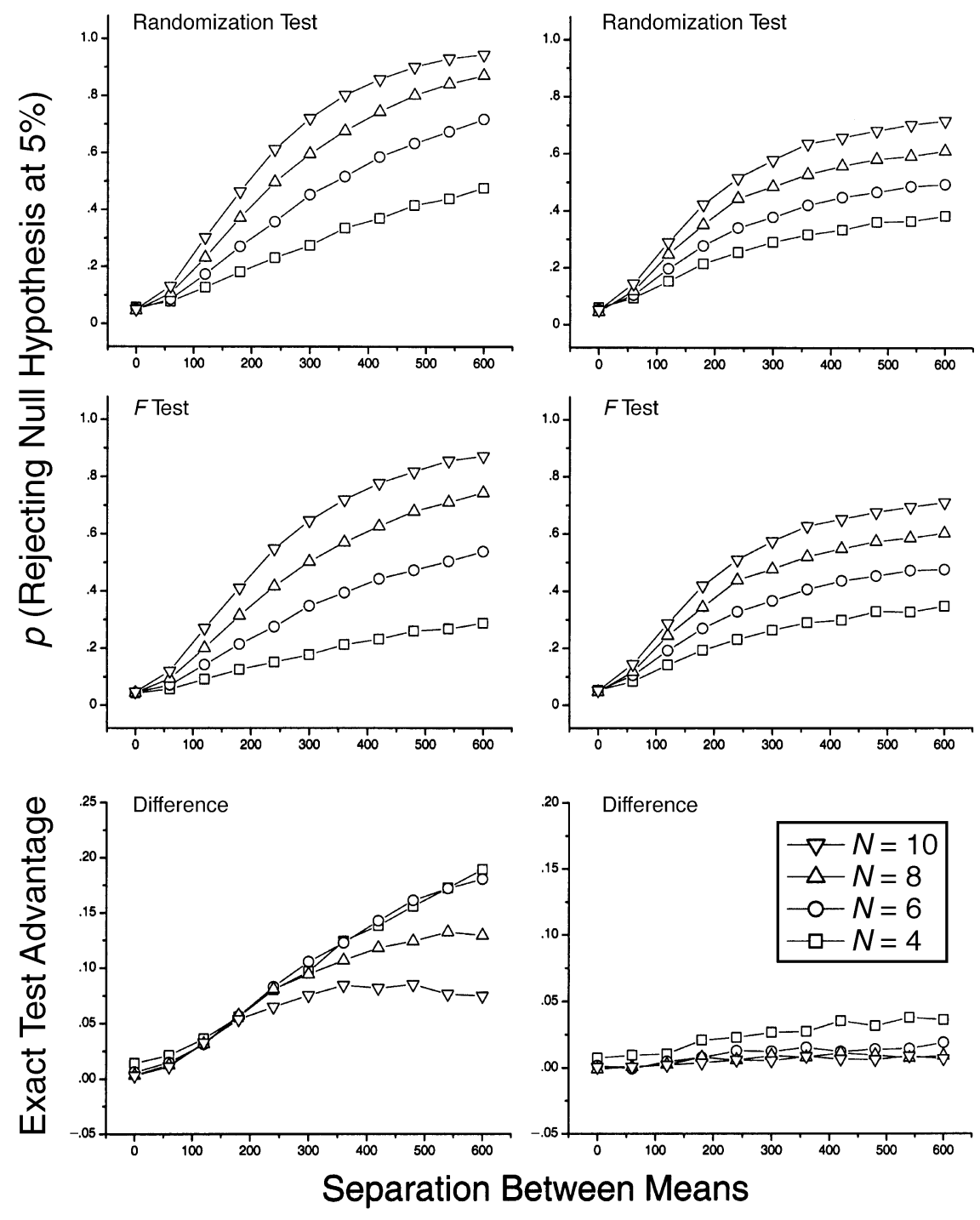

Figure 3. Monte Carlo comparison of the between-subjects randomization test and the $F$ test with skewed error; the skew is correlated with the treatment. The top and middle panels of the left column show the probability of rejecting the null hypothesis at the $5 \%$ level of confidence as a function of the number of observations per cell and effect size for the randomization and $F$ tests, respectively. The bottom panel of the left column shows the difference between the two tests. The right column shows a control comparison using Gaussian error with the variance yoked to the skewed examples shown in the corresponding panels of the left column. 
in studies of memory and choice - the advantage favoring the randomization test was as large as $20 \%$.

Variance (not skew) correlated with the treatment. As I noted earlier, one consequence of skewing the error distribution is to increase its variance. To consider the effect of the increase in variance independent of skew, I repeated the analysis but used Gaussian error, with variance correlated with the treatment. To do so, I set the standard deviation of the experimental cell equal to that of the corresponding cell of the skewed case; that is, variance in the Gaussian case for the experimental cell was set to the variance of the corresponding ex-Gaussian case (i.e., $\operatorname{sigma}^{2}+t a u^{2}$ ). Finally, I manipulated the separation between the means of the two cells by adding a constant to the experimental cell, the same manipulation used in the data reported in Figure 1.

The top panels in the right column of Figure 3 show that both tests rejected a true null hypothesis about 5\% of the time. Increasing both the separation between the means and the number of observations per cell increased the probability of detecting the difference. As shown in the bottom right panel, the large and systematic advantage favoring the randomization test was lost, a sharp contrast with when skew was correlated with the treatment effect. Hence, the systematic advantage shown when the treatment effect is correlated with skew is a consequence of skew, not of the increase in variance.

A final aspect of the data in Figure 3 deserves note. Although the systematic advantage favoring the randomization test largely disappeared with normal error, the sensitivity of both the randomization and $F$ tests was decreased in relation to the corresponding skewed example. The decrease is paradoxical because the variance was equated across the two cases.

\section{Extensions to Other Designs}

The design considered, so far, compared two independent groups of subjects. In within-subjects designs, each subject is tested twice, and the experimenter randomly assigns the conditions to the successive tests. The comparison of interest lies in the difference between the conditions for each subject. Although the fundamental logic of the randomization test is the same, the chance model is changed, because the two measurements are correlated.

If the treatment has no real effect, any difference between the means of the two cells must reflect test-to-test, or time-to-time, variability between the pairs of observations. Hence, the difference for a given subject is a matter of chance, and the sum of differences across subjects represents one of a set of possible sums defined by the combination of differences. If there are $M$ subjects, there are $2^{M}$ possible sums of differences, and if the treatment has no real effect, all of the sums are equally likely. To compute a test for significance, the experimenter counts the number of possible outcomes that would yield a sum as extreme as that actually obtained. Table 1 presents a simple recursive algorithm to carry out the calculations.

The within-subjects design tells the same story as the between-subjects case documented in Figures 1-3. In between- and within-subjects designs, the randomization test works best when error is normal. Provided that the number of combinations is large enough, when error is skewed, the randomization test is more sensitive than the corresponding $F$ test is, and if skew is correlated with the treatment, the advantage for the randomization test grows with the treatment. To document the sensitivity advantage with the within-subjects randomization test, Figure 4 presents data using skewed error with the amount of skew correlated with the treatment (data that corre-

Table 1

An F90 Routine to Permute the Sign of Differences for the Within-Subjects Randomization Test

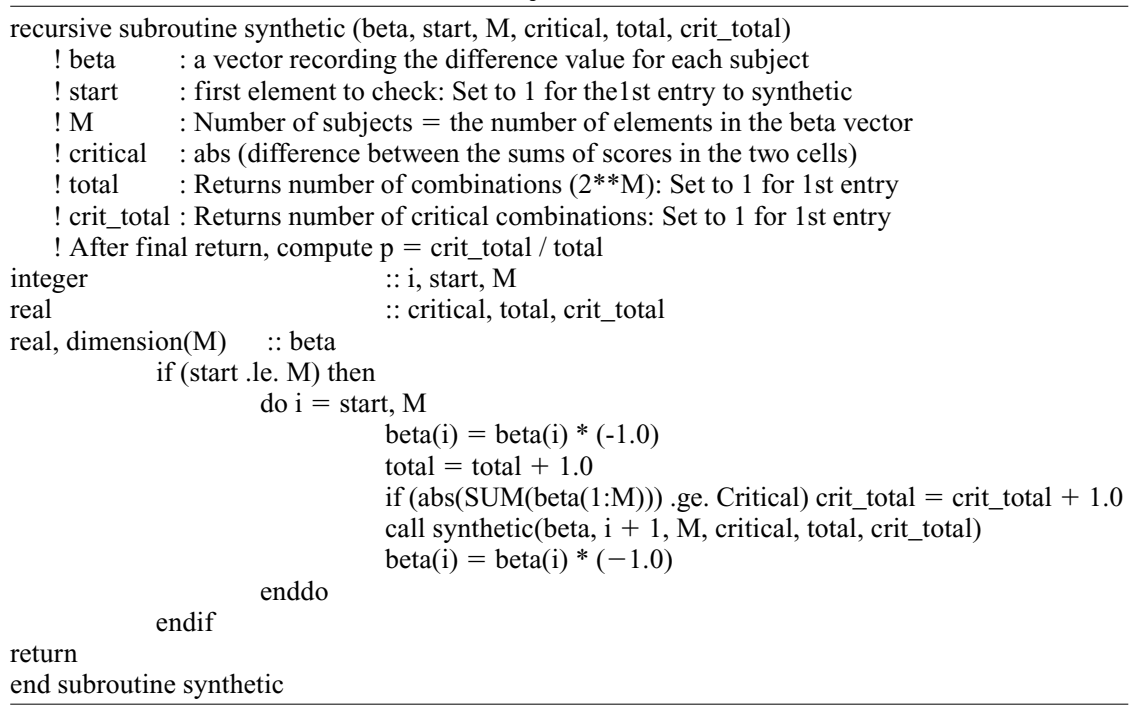




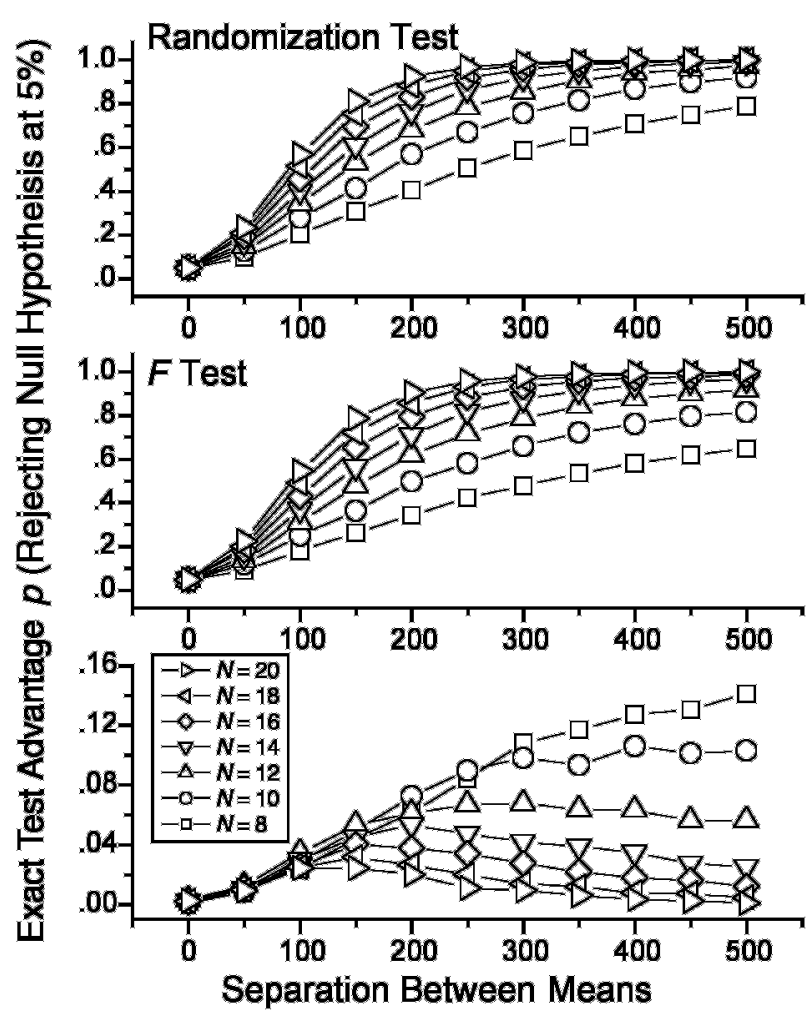

Figure 4. Monte Carlo comparison of the within-subjects randomization test with the $F$ test, using skewed error; the skew is correlated with the treatment. The top and middle panels show the probability of rejecting the null hypothesis at the $5 \%$ level of confidence as a function of the number of observations per cell and effect size for the randomization and $F$ tests, respectively. The bottom panel shows the difference between the two tests.

spond to the between-subjects case shown in the left panel of Figure 3). For the analysis, I set $m u=400$, sigma $=50$, and tau $=80$. I manipulated the separation between the means by increasing tau in steps of 50; that is, I manipulated the separation by increasing the skew in the experimental cell, the same manipulation reported for the between-subjects design in Figure 3.

As shown in the top two panels of Figure 4, both the randomization and the $F$ tests rejected a true null hypothesis about $5 \%$ of the time, and increasing both the separation between the means and the number of observations per cell increased the probability of detecting the difference. As in the corresponding between-subjects design, a large and systematic advantage favored the randomization test over the $F$ test (shown in the bottom panel of Figure 4). In short, for both between- and withinsubjects designs, in cases where the skew is correlated with the treatment, which are typical of studies of memory and choice, the advantage favoring the randomization test was both large and correlated with the skew.

There are two important qualifications to the generality of the present results. (1) The treatment effect was always in the direction of the skew. The choice was made deliberately because it matches the empirical situation in a wide range of ongoing research using RT, but the results may not extend to cases in which skew is not in the direction of the treatment. (2) The cells were always equal. It is possible that the advantage for exact tests over the $F$ test may change, depending on the relative number of observations in the cells. Both qualifications should be topics of future research.

\section{CONCLUSIONS}

Assigning subjects to cells defines the chance model on which the randomization test is based. Hence, if an experiment has been conducted properly, the randomization test can be justified without reference to distribution assumptions. That said, it is clear that the randomization test works best when the underlying distribution of error has the properties that Gauss built into his account of measurement error: Small errors are more frequent than large ones, and the distribution of errors is symmetrical so that the mean of the measurements is not biased systematically.

If the number of observations per cell is large enough, when error takes a normal form, the randomization test and the corresponding $F$ test are equivalent. When error is skewed, however, the randomization test is never less sensitive than the $F$ test, and, if the skew is correlated with the treatment, the randomization test is systematically more sensitive than the corresponding $F$ test. Experimenters who use skewed dependent measures, such as swimming time in the water maze, may find the additional sensitivity a considerable advantage.

The functional equivalence of the randomization test and the $F$ test with normal error hides a controversial issue. The randomization test depends on a chance model derived from random distribution of subjects to conditions at the time the experiment was conducted. The $F$ test depends on a chance model derived in terms of random sampling from a normally distributed population. Random sampling allows one to express the test for significance as an estimation problem - that is, as a way to estimate parameters of a defined population. Ludbrook and Dudley (1998) have questioned whether the estimation approach is appropriate for biomedical sciences. They surveyed research in the field and noted that experimenters rely more frequently on random distribution of subjects to cells than on random sampling from defined populations. Consequently, because the chance model underlying the randomization test maps more closely on current practice than do models based on random sampling, they argued that it should be preferred to the $F$ test (their argument discounts the Gauss theory of error as a justification for using a sampling model). As Manly (1997) notes, the definition of a population is often so broad that random sampling is an artificial possibility. Suppose, for example, an experimenter takes 20year-old volunteers from an undergraduate course and conducts an experiment after randomly splitting them 
into two groups. A randomization test on the resulting data would be valid and would speak to whether the difference in means reflects the treatment or the distribution of differences among the subjects to the two cells. A conclusion about 20-year-old students in general, however, would be valid only on the assumption that the subjects are equivalent to a random sample of all 20year-old students. Unfortunately, the population of 20 year-old students is so broad as to call into question the practicality of random sampling. Instead, Manly suggests, "in the absence of truly random samples, convincing evidence of an effect requires that it be demonstrated consistently at different times and in different places" (p. 3). Nevertheless, random sampling from a defined population followed by random assignment clearly should support a stronger generalization than random assignment alone, and following the precedent established by Edgington (1995) and Kempthorne and Doerfler (1969), Manly recommends that the term permutation test be reserved for the first case with the term randomization test reserved for the second.

The philosophical underpinnings of statistical inference are beyond the scope of the present article. Nevertheless, random assignment is a prerequisite for causal inference, regardless of how the subjects are obtained. Fisher (1959) distinguished true sampling of cases from an identifiable population, as in a quality-control or acceptance procedure, from the use of statistical sampling as a metaphor justified by the Gauss theory of error in the analysis of experiments. In his words, "the only populations that can be referred to in a test for significance have no objective reality" (p. 78). Rather, they refer to distribution of error or "uncontrolled variability . . . in the physical or biological material under examination" (p. 76). At least for Fisher, then, the test for significance in the analysis of a comparative experiment addresses the procedure used in the experiment; it is not an exercise in estimation parallel to a census or political survey. Fisher's emphasis on procedure echoes his view concerning the role of experimentation in science: "A phenomenon is experimentally demonstrable when we know how to conduct an experiment which will rarely fail to give us a statistically significant result" (Fisher, 1935/1966, p. 14).

The logical foundation of the randomization test also raises a technical issue. The $F$ test tests the null hypothesis that the population means are equal. Because a randomization test does not pretend to estimate population parameters, it cannot, therefore, have the same null hypothesis as the $F$ test. The null hypothesis appropriate to the randomization concerns equality of distributions produced after the fact-it is a direct answer to a procedural question about potential confounding subject differences with the treatment when the subjects were assigned to cells.

Finally, the present results point to three additional issues. The first is a question for statistical theory. To consider the consequences of violating an assumption required in the derivation of a statistical test, it is standard practice to compare the test when its assumptions are true against the case when they have been violated. As the present examples illustrate, a sensible alternative is to compare a test when its assumptions have been violated with a randomization test. The randomization test provides a plausible real-world comparison, because its validity depends on random assignment instead of a specific distribution of error.

A second issue concerns the practical alternatives available to researchers. When faced with likely violation of normal-error assumptions, many researchers adopt tests based on ranks, on the principle that they are easy to compute and appropriately conservative. Unfortunately, considerable information is lost when raw data are converted to ranks. A rank transformation does not preserve the information needed, for example, to distinguish the two examples of skew documented by Hockley (1984). Moreover, as Mundry and Fisher (1998) note, because rank tests are not uniformly well implemented in popular statistical packages for computers, they are often not appropriately conservative. In particular, critical $p$ values are often miscalculated for small $n$ examples. The randomization test provides a sensible alternative that preserves the information available in the data. But, as Onghena and May (1995) note, it is often tricky to implement a randomization test correctly using standard computer packages.

The final issue concerns the economics of experimentation. Although we can anticipate that error will be skewed using measures based on time, it is difficult to anticipate whether a manipulation will increase skew (as Hockley, 1984, found in a recognition-memory task) or will translate the distribution (as Hockley found in a visual-search task). If error is skewed-especially if the treatment effect is correlated with the degree of skewthe randomization test is systematically more sensitive than the standard normal-error tests. Hence, there is a good chance that an $F$ test will fail to detect effects that could have been found using a randomization test. Because the cost of computing has decreased dramatically over the last decade, it is now smaller than the cost of conducting the experiment. Hence, the randomization test is now an increasingly practical alternative to tests based on normal-distribution theory.

\section{REFERENCES}

Ashby, F. G., Tein, J. Y., \& Balakrishnan, J. D. (1993). Response time distributions in memory scanning. Journal of Mathematical Psychology, 37, 526-555.

BAKER, F. B., \& COLLIER, R. O. (1966). Some empirical results on variance ratios under permutation in the completely randomized design. Journal of the American Statistical Association, 61, 813-820.

Box, G. E. P., \& Andersen, S. L. (1955). Permutation theory in the derivation of robust criteria and the study of departures from assumption. Journal of the Royal Statistical Society: Series B, 17, 1-34.

Bradley, J. V. (1968). Distribution-free statistical tests. Englewood Cliffs, NJ: Prentice-Hall.

Edgington, E. S. (1995). Randomization tests (3rd ed.). New York: Dekker.

FISHER, R. A. (1959). Statistical methods and scientific inference (2nd ed.). Edinburgh: Oliver \& Boyd. 
FISHER, R. A. (1966). Design of experiments (8th ed.). Edinburgh: Oliver \& Boyd. (Original work published 1935)

Freedman, D., Pisani, R., Purves, R., \& Adhikari, A. (1991). Statistics (2nd ed.). New York: Norton.

Heathcote, A., Brown, S., \& Mewhort, D. J. K. (2002). Quantile maximum likelihood estimation of response time distributions. Psychonomic Bulletin \& Review, 9, 394-401.

Heathcote, A., Popiel, S. J., \& Mewhort, D. J. K. (1991). Analysis of response time distributions: An example using the Stroop task. Psychological Bulletin, 109, 340-347.

HockLEy, W. E. (1984). Analysis of response time distributions in the study of cognitive processes. Journal of Experimental Psychology: Learning, Memory, \& Cognition, 10, 598-615.

HoEfFDing, W. (1952). The large-sample power of tests based on permutations of observations. Annals of Mathematical Statistics, 23, 169-192.

Keller-McNulty, S., \& Higgins, J. J. (1987). Effect of tail weight and outliers on power and type-one error of robust permutation tests for location. Communications in Statistics: Simulation \& Computation, 16, 17-35.

Kempthorne, O., \& Doerfler, T. E. (1969). The behaviour of some significance tests under experimental randomization. Biometrika, 56, 231-248.

LINK, S. W. (1990). Modeling imageless thought: The relative judgment theory of numerical comparison. Journal of Mathematical Psychology, 34, 2-41.

LudBRooK, J., \& Dudley, H. (1998). Why permutation tests are superior to $t$ and $F$ tests in biomedical research. American Statistician, 52, 127-132.

Manly, B. F. J. (1997). Randomization, bootstrap and Monte Carlo methods in biology (2nd ed.). London: Chapman \& Hall.

Moyer, R. S., \& Landauer, T. K. (1967). Time required for judgements of numerical inequality. Nature, 215, 1519-1520.

MundRY, R. (1999). Testing related samples with missing values: A permutation approach. Animal Behaviour, 58, 1143-1153.

MUNDRY, R., \& FISCHER, J. (1998). Use of statistical programs for non- parametric tests of small samples often leads to incorrect $P$ values: Examples from Animal Behaviour. Animal Behaviour, 56, 256-259.

Nichols, T. E., \& Holmes, A. P. (2001). Nonparametric permutation tests for functional neuroimaging: A primer with examples. Human Brain Mapping, 15, 1-25.

Onghena, P., \& May, R. B. (1995). Pitfalls in computing and interpreting randomization test $p$ values: A commentary on Chen and Dunlap. Behavior Research Methods, Instruments, \& Computers, 27, 408-411.

Pitman, E. J. G. (1937). Significance tests which may be applied to samples from any population. Supplement to the Journal of the Royal Statistical Society, 4, 119-130.

Press, W. H., Teunolsky, S. A., Vetterling, W. T., \& Flannery, B. P. (1992). Numerical recipes in FORTRAN: The art of scientific computing (2nd ed.). New York: Cambridge University Press.

RATCLIFF, R., \& MuRdock, B. B. (1976). Retrieval processes in recognition memory. Psychological Review, 83, 190-214.

Romano, J. P. (1990). On the behavior of randomization tests without a group invariance assumption. Journal of the American Statistical Association, 85, 686-692.

Smith, D. G., \& Mewhort, D. J. K. (1998). The distribution of latencies constrains theories of decision time: A test of the random-walk model using numeric comparison. Australian Journal of Psychology, 50, $149-156$.

SternberG, S. (1969). Memory scanning: Mental processes revealed by reaction-time studies. American Scientist, 57, 421-457.

STIGLER, S. M. (1986). The history of statistics: The measurement of uncertainty before 1900. Cambridge, MA: Harvard University Press.

\section{NOTE}

1. The number of combinations of $M$ objects taken $r$ at a time, $\mathrm{C}(M, r)$, is given by $M ! /(r !(M-r) !)$.

(Manuscript received January 18, 2004;

revision accepted for publication August 14, 2004.) 